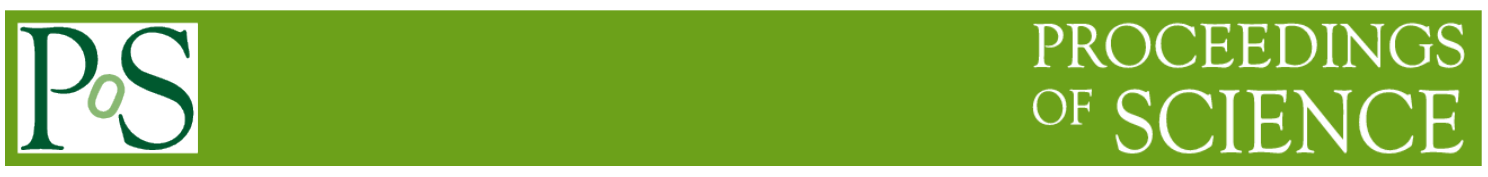

\title{
The Wearable Level for Wearable Devices
}

\section{Yunting $\mathbf{L i}^{1}$}

China Electronic Product Reliability and Environment Testing and Research Institute Guangzhou,510610, China

\section{Fan Zhang ${ }^{2}$}

China Electronic Product Reliability and Environment Testing and Research Institute Guangzhou,510610, China

E-mail: zhangfan@ceprei.com

\section{Chunhui Yang, Dongyu Yang}

China Electronic Product Reliability and Environment Testing and Research Institute Guangzhou,510610, China

\begin{abstract}
With the development of smart mobile computer, more and more portable devices are coming into market that includes wearable devices. To evaluate the wearable devices, the wearable level is an important reference. In this paper, we proposed to evaluate wearable devices based on quality characteristics. However, the characteristics are usually unstable, historical sample size is small, index, show obvious non-normal distribution. These features make it hard to use the general statistical techniques for effective evaluation. A model based on support vector machine (SVM) is proposed to distinguish the wearable level. This paper introduces the current situation of wearable devices, descripts its classification and evaluation principle, and gives out the classification model and algorithm for wearable level. This work can be a beneficial reference to wearable standard.
\end{abstract}

CENet2015

12-13 September 2015

Shanghai, China

\footnotetext{
${ }^{1}$ Speaker

${ }^{2}$ Coresponding auther
} 


\section{Introduction}

Wearable devices are mobile intelligent devices, which integrate new generation of information technology with the wireless communication, cloud services and big data to perception, recognition and interaction with user. It helps to realize portable entertainment health and production, etc.

In January 2014 at the annual meeting of the world's consumer electronics show in Las Vegas, wearable devices, many of the world's best-known consumer electronics industry upstream and downstream enterprises are turning to the wearable devices [1]. It is shown important research value and application potential in the industrial, medical, military, education, entertainment, and many other areas [2,3]. Effective evaluation method is needed as soon as possible to support the standards of wearable devices and guide the progress of product development [4].

This paper is focused on evaluating the wearable level of wearable devices with quality characteristic. It shows an evaluation model called the wearable level evaluation method. The method is briefly described in following. For testing the method, a simulations experiment on SPSS has been done.

In this context, our paper is organized as follows. In Section 2, we make a suggestion for the classification of wearable devices that cites General Wellness: Policy for Low Risk [5]. Section 3 describes the quality characteristics of wearable devices. The quality characteristics are defined as the capability to meet the requirements of explicit and implicit function. Section 4 introduces the wearable level. We describe the evaluation method and present the classification model and algorithm of wearable devices, and then we conclude with summary remarks in Section VI.

\section{Wearable Devices Classification}

The classification of the wearable devices is suggested to the main application field of products. According to the application field, wearable device can be mainly divided into: consumer electronics and medical health.

Consumer electronics class is basically considered as low risk general products. A general wellness product, for the purposes of this guidance, has an intended use that relates to a maintaining or encouraging a general state of health or a healthy activity, or an intended use claim that associates the role of healthy lifestyle with helping to reduce the risk or impact of certain chronic diseases or conditions and where it is well understood and accepted that healthy lifestyle choices may play an important role in health outcomes for the disease or condition.

Medical health class is basically considered as medical equipment which directly or indirectly for human instrument, equipment, instruments, in vitro diagnostic reagents and calibration, materials, and other similar or related items, including computer software that is needed.

Wearable device is not only a kind of hardware equipment, but also can interact through software and support data exchange, and the cloud to achieve powerful features, such as helping users to sports and health management [6]. It monitors information of users every day, such as sleep time, movement distance data consumption quantity of heat, diet, etc. Data can be transmitted to the smartphone APP or the cloud through the USB port, or wireless Bluetooth technology. After professional data statistical analysis, to the users receive feedback guidance.

\section{The Quality Characteristics of Wearable Devices}

Wearable devices have very broad product category and different category has its own features. Standards set a threshold for the production. Lower specification level may reduce the trust of consumers. However, higher specification level may hinder technology innovation. 
Standards, therefore, should be in the relationship between enterprises and consumers to achieve a dynamic balance, and guide the orderly and healthy development of wearable computing industry, at the same time form the influence in the consumer groups [7].

\subsection{Quality Characteristics}

The quality characteristics of wearable devices are the capability to meet the requirements of explicit and implicit function. Quality characteristics could think as the basis of evaluation, though widely, but not all product design, manufacturing, sales and other related features are quality features. According to the quality and the quality definition, we know that quality is an inherent property of products. If there is nothing to do with the product or not itself inherent characteristics, it is not quality. Such as product prices, product processing precision, machine tool cutting parameters and so on.

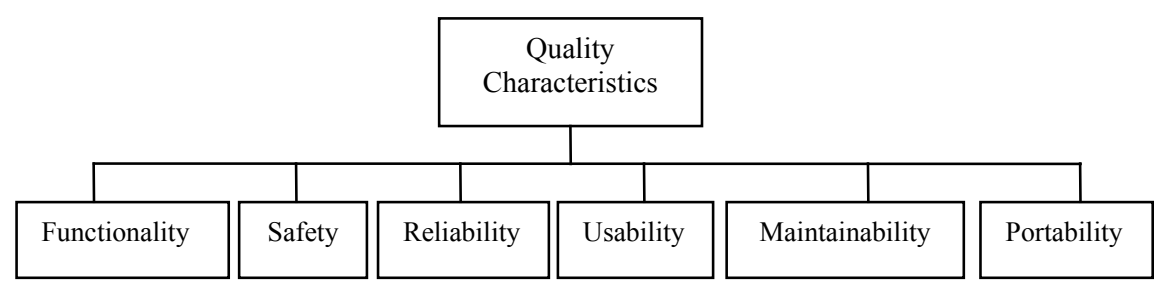

Figure 1: the quality charicteristic model of wearable devices

Wearable devices are suggested to consider quality characteristics as evaluation standard [s1]. We proposed a structure in figure 1. The quality characteristics of wearable devices consist of functionality, safety, reliability, usability, maintainability and portability.

Functionality: when under the specified conditions, wearable devices provide the capability to meet the requirements of explicit and implicit function. This feature is related to what to do to meet the requirements, and other characteristics are mainly related to when to meet the requirements and how to meet the requirements.

Safety: the ability to protect health, information, data and privacy. Material and function are safe to user. Unauthorized personnel or system can't read or modify the information and data, and not declined to authorized personnel or system access to them.

Reliability: the ability to maintain a prescribed level of performance under the specified conditions when using. The reliability of the limitations is due to fault of the requirements, design, and implementation. This definition has higher requirement than "complete the required function".

Usability: the ability to attract users by understanding and learning under specified conditions. This characteristic is a subjective probability. Some aspects of functionality, reliability and efficiency will affect the ease of use, but cannot replace usability.

Maintainability: the ability to avoid unexpected results caused by modifies the software or hardware. Modification may include corrective or improvement.

Portability: the ability to transplant software from an environmental to another environment. The environment may include organization, hardware or software environment. 


\subsection{The feature Metrics}

This section gives a discussion about the feature related to different category for the further description about quality characteristic, for example, smart band and smart watch, etc. We list the features of smart band according to its quality characteristic in Table1.

\begin{tabular}{|l|l|l|l|l|l|l|}
\hline & Functionality & Safety & Reliability & Usability & $\begin{array}{l}\text { Maintainab } \\
\text { ility }\end{array}$ & Portability \\
\hline \multirow{3}{*}{$\begin{array}{l}\text { smart } \\
\text { band }\end{array}$} & $\begin{array}{l}\text { Accuracy } \\
\text { metrics }\end{array}$ & $\begin{array}{l}\text { Material } \\
\text { safety } \\
\text { metrics }\end{array}$ & $\begin{array}{l}\text { Maturity } \\
\text { metrics }\end{array}$ & $\begin{array}{l}\text { Understan } \\
\text { dability } \\
\text { metrics }\end{array}$ & $\begin{array}{l}\text { Changeabil } \\
\text { ity metrics }\end{array}$ & $\begin{array}{l}\text { Adaptabilit } \\
\text { y metrics }\end{array}$ \\
\cline { 2 - 7 } & y metrics & $\begin{array}{l}\text { Function } \\
\text { safety }\end{array}$ & $\begin{array}{l}\text { fault } \\
\text { tolerance } \\
\text { metrics }\end{array}$ & $\begin{array}{l}\text { Learnabili } \\
\text { ty metrics }\end{array}$ & $\begin{array}{l}\text { Stability } \\
\text { metrics }\end{array}$ & $\begin{array}{l}\text { Installabilit } \\
\text { y metrics }\end{array}$ \\
\cline { 2 - 7 } & $\begin{array}{l}\text { Compatibility } \\
\text { metrics }\end{array}$ & $\begin{array}{l}\text { Informatio } \\
\text { n safety }\end{array}$ & $\begin{array}{l}\text { Recoverabilit } \\
\text { y metrics }\end{array}$ & $\begin{array}{l}\text { Operabilit } \\
\text { y metrics }\end{array}$ & $\begin{array}{l}\text { Testability } \\
\text { metrics }\end{array}$ & $\begin{array}{l}\text { Co- } \\
\text { existence } \\
\text { metrics }\end{array}$ \\
\hline
\end{tabular}

Table 1: the feature metrics for wearable devices

The column is different category of wearable devices, and the row shows quality characteristic which use feature metrics. For different category, we can measure and calculate the feature metrics in table 1, quantified it, and then use the result of normalization as the input of evaluation model.

\section{The Wearable Level}

The evaluation of wearable level is considered as a classification problem. Because different users have different understanding to quality characteristics, there is inconsistency problem. In order to solve this problem, we proposed a rating model. This section describes the rating model of wearable level, which based on optimization technique through processing quality characteristics.

\subsection{Evaluation Method}

Existing evaluation method is aimed at single attribute of wearable devices. On the one hand, the objective directivity of evaluation results is not strong. the findings point is fuzzy and cannot give out the comprehensive evaluation results for a reasonable planning; on the other hand, the evaluation results is just a comparison provided for the user's reference, which lack of calculation method and corresponding reference. Wearable device itself is a system, which the evaluation of the situation was complex and involves a lot. Until now, there is still not a unified evaluation method. In order to achieve the wearable devices in an objective and fair, accurate and reliable evaluation purpose,

We should follow some basic principles: (1) The evaluation method should consider the characteristics of wearable devices; (2) The evaluation method should concerns about the dynamic development of wearable devices; (3) The evaluation method should reflect common property and avoid single characteristic factor to cause high rating.

To achieve this, we consider using quality characteristics as the input of evaluation method. A quality is an 'inherent or distinguishing characteristic, a property, or a personal trait'. Quality denotes the character, disposition, or nature of something. A characteristic is 'a feature that helps distinguish a person or thing, a distinguishing mark or trait'. Quality is slightly more inclusive than characteristic. Characteristic's meaning is more about a distinction. Each characteristic is distinguishing feature of the product (service).

The output wearable level is shown in figure 2. No.1 is high level that means better comprehensive evaluation. No.2 is middle level, and No.3 is low level that means lower quality or user experience. 


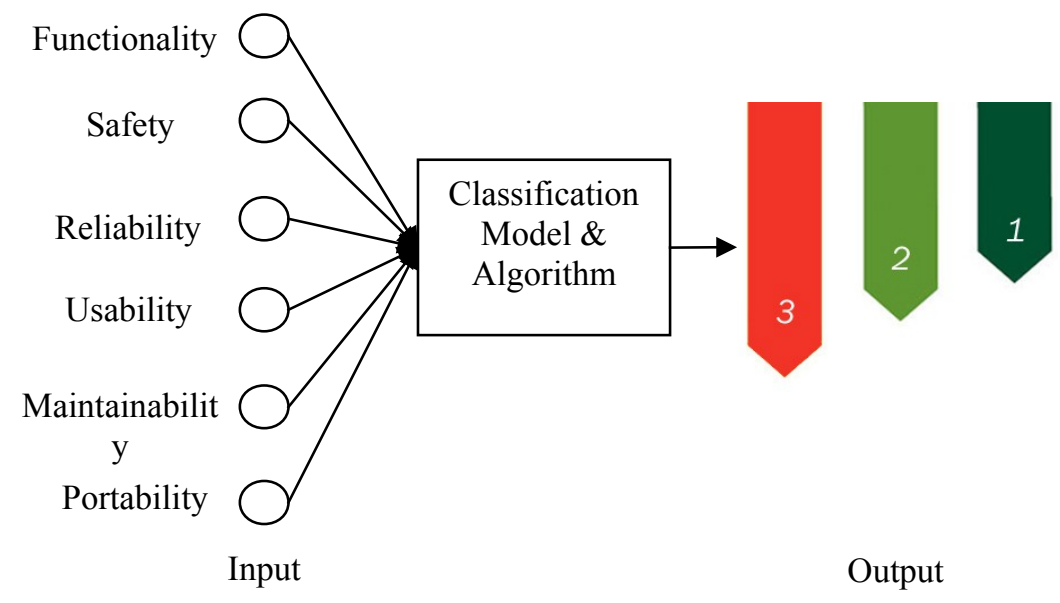

Figure 2: The 3 level for wearable devices

\subsection{Classification Model \& Algorithm}

IBM SPSS Modeler offers a variety of modeling methods taken from machine learning, artificial intelligence, and statistics. The methods available on the Modeling palette allow designer to derive new information from data and to develop predictive models. Each method has certain strengths and is best suited for particular types of problems. We proposed our classification model based on IBM SPSS Modeler, and used SVM algorithm [8].

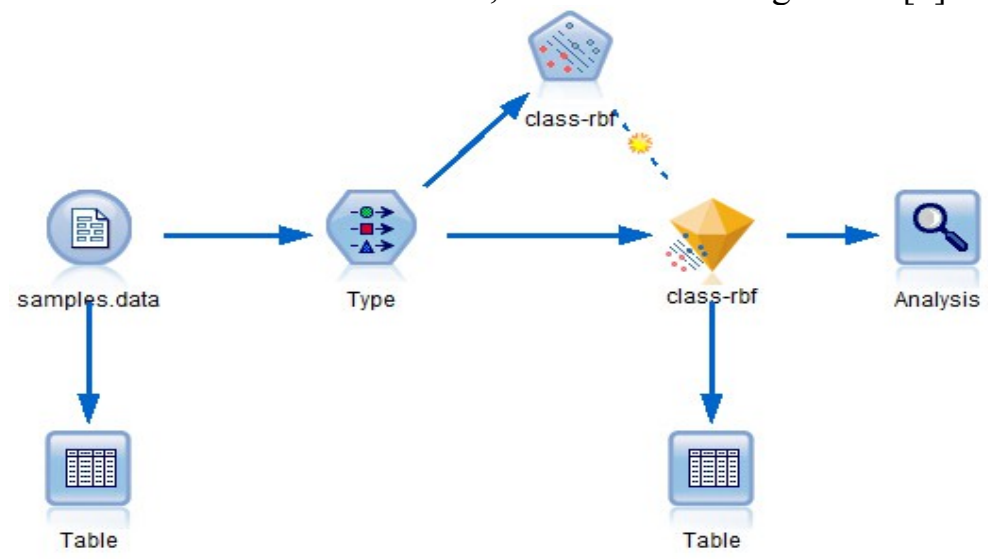

Figure 3: SVM model for classification

Support Vector Machine (SVM) is currently a hot topic in the machine learning community, creating a similar enthusiasm at the moment as Artificial Neural Networks used to do before $[9,10]$. SVMs yet represent a powerful technique for general (nonlinear) classification. SVM is a robust classification and regression technique that maximizes the predictive accuracy of a model without overfitting the training data [11].

For classification about the training set $\mathrm{T}$

$$
T=\left\{\left(x_{1}, y_{1}\right), \cdots,\left(x_{l}, y_{l}\right)\right\} \in(x \times y)^{l}
$$

Where $x_{i} \in x=R^{n}, y_{i} \in\{-1,1\}$.

The goal is to find an optimal function $g(x), \quad x \in R^{n}$ to get finally decision function

$$
f(x)=\operatorname{sgn}(g(x))
$$

The (4.2) is restricted to the linear case. For quality characteristics, it's nonlinear. Here, we use the decision function like (4.3). 


$$
f(x)=\operatorname{sgn}\left(\sum_{(i=1)}^{l} y_{i} \alpha_{i} K\left(x_{i}, x\right)+b\right)
$$

Where $b=y_{j}-\sum_{(i=1)}^{l} y_{i} \alpha_{i} K\left(x_{i}, x_{j}\right) j \in\left\{j \mid 0<\alpha_{j}<C\right\}, \quad K\left(x_{i}, x\right)$ is the kernel function called Radial Basis Function (RBF).

$$
K\left(x, x_{i}\right)=\exp \left\{\frac{-\left|x-x_{i}^{2}\right|}{\sigma^{2}}\right\}
$$

Classification results of wearable level are obtained by according to (4.1).

\section{Conclusion}

In this paper, we proposed a quality characteristic based evaluation method for wearable devices, which considers user experience as an important quality characteristic. This work is useful for standard setting, since there are still not wearable standard in the market. We created the feature metrics table and use it as the input parameters of classification model, and the output is wearable level. After descripting the quality characteristics, we give a classification model based on SVM, which can process data from measurement efficiently. Remarkably, SVM works by mapping the data of quality characteristics to a high-dimensional feature space so that quality characteristics points can be categorized. Analysis shows that SVM is a good choice for classification both in nonlinear and multiclass classification. In the future work, wearable level standard is under our consideration. In addition, the design and experiment of feature metrics is also interesting.

\section{References}

[1] Hong Dai. Wearable intelligent technical product and standard[J]. China Standard, 1 (z1): 021(2013). (In Chinese) .

[2] Nanxiang Yu, Dongyi Chen, HIAHOU Shiji. The application and new development in wearable computing [J]. Digital Communication, 39(4):13-20(2012),..

[3] Zhizhong Zhao,Yi Xu. The New development of wearable computing devices[J]. Science \& Technology for China's Mass Media, 4(11): 123-125(2013). (In Chinese)

[4] David Kotz, Ryan Halter, Cory Cornelius, Jacob Sorber, Minho Shin,Ronald Peterson, Wearable computing device for secure control of physiological sensors and medical devices, with secure storage of medical records, and bioimpedance biometric: U.S. Patent Application 14/312,316[P]. 2014-6-23.

[5] U.S.Food and Drug Administration. Draft Guidance for Industry and Food and Drug Administration Staff, General Wellness:Policy for Low Risk Devices,

http://www.fda.gov/downloads/medicaldevices/deviceregulationandguidance/guidancedocuments/uc m429674.pdf

[6] Bo Pang, Lillian Lee. Seeing stars: Exploiting class relationships for sentiment categorization with respect to rating scales [C]. Proceedings of the 43rd Annual Meeting on Association for Computational Linguistics. Association for Computational Linguistics, 1(1): 115-124 (2005).

[7] Wei Liang. On Effective Implementation of Alliance Standard[J]. Standard Science, 44(9): 014016 (2013).

[8] McCormick, Keith; Abbott, Dean. IBM SPSS Modeler Cookbook[M]. Packt Publishing, Birmingham, 382p (2013).

[9] Shibuya N, Nukala B T, Rodriguez A I, et al. A real-time fall detection system using a wearable gait analysis sensor and a Support Vector Machine (SVM) classifier[C], 2015 Eighth International Conference on Mobile Computing and Ubiquitous Networking (ICMU). IEEE Computer Society, $1(1)$ : 66-67 (2015). 
[10] Meyer D, Wien F H T. Support vector machines $[\mathrm{J}]$. The Interface to libsvm in package e1071, 1(1): 469-524 (2014).

[11] Kaur H, Singh B. Classification and grading rice using multi-class SVM[J]. International Journal of Scientific and Research Publications, 3(4): 035-042 (2013). 